\title{
Radiographic and Ultrasonographic Findings in Three Surgically Confirmed Cases of Small Intestinal Ischemia Related to Mesenteric Volvulus or Intestinal Torsion in Dogs
}

\author{
Elina Rautala', Pia Björkenheim ${ }^{2}$, Merja Laitinen ${ }^{3}$ \\ ${ }^{1}$ Department of Equine and Small Animal Medicine, Diagnostic Imaging, University of Helsinki, Helsinki, Finland \\ ${ }^{2}$ Department of Equine and Small Animal Medicine, Surgery, University of Helsinki, Helsinki, Finland \\ ${ }^{3}$ St. John's Innovation Centre, Cambridge, UK \\ Email: elina.tapio@helsinki.fi
}

How to cite this paper: Rautala, E., Björkenheim, P. and Laitinen, M. (2017) Radiographic and Ultrasonographic Findings in Three Surgically Confirmed Cases of Small Intestinal Ischemia Related to Mesenteric Volvulus or Intestinal Torsion in Dogs. Open Journal of Veterinary Medicine, 7, 99-110.

https://doi.org/10.4236/ojvm.2017.79010

Received: July 25, 2017

Accepted: September 12, 2017

Published: September 15, 2017

Copyright $\odot 2017$ by authors and Scientific Research Publishing Inc. This work is licensed under the Creative Commons Attribution International License (CC BY 4.0).

http://creativecommons.org/licenses/by/4.0/

\section{(c) (i) Open Access}

\begin{abstract}
This case report describes the radiographic and ultrasonographic findings of three surgically confirmed cases of mesenteric volvulus or intestinal torsion in dogs. In all three cases, ultrasonographic findings included segmental ileus and absent or markedly reduced peristalsis of the affected small intestine, and partial loss of wall layering with increased overall echogenicity of the intestinal wall, but with normal to mildly increased wall thickening. No blood flow was detected in the affected small intestinal wall when assessed with colour Doppler. A moderate amount of peritoneal effusion was also detected with hyperechoic omental and mesenteric fat tissue throughout the peritoneal cavity. Few reports describe ultrasonographic findings of small intestinal ischemia in small animals. In all three cases presented here, ultrasound was helpful in demonstrating typical intestinal wall changes and helped to obtain the correct diagnosis of ischemic disease of the small intestine.
\end{abstract}

\section{Keywords}

Mesenteric Volvulus, Intestinal Volvulus, Dog, Ultrasonography, Radiography

\section{Introduction}

Mesenteric volvulus or intestinal torsion is a rare cause of acute abdomen. The condition is life-threatening and requires rapid stabilization of the patient and surgical intervention. Mesenteric or intestinal volvulus is defined as twisting of the intestine on its mesenteric attachment [1]. Sometimes the term mesenteric 
torsion is also used. In intestinal torsion, the intestine rotates around itself on its long axis [2]. Intestinal ischemia is caused by occlusion of the cranial mesenteric artery and vein leading to gas and fluid accumulation into the intestinal lumen, oedema and necrosis of the intestinal wall, disruption of the mucosal barrier, toxin release and translocation of bacteria eventually resulting septic peritonitis and shock [1]. Symptoms are usually acute although a case of chronic mesenteric volvulus has been reported in a dog [3] [4] [5]. The common clinical signs include vomiting, hematochezia, abdominal pain and distension, and a rapid development of shock [3] [4]. Large breed dogs, particularly German shepherd dogs, are predisposed to mesenteric volvulus, but it has also been reported in small dogs and cats [3] [4] [6] [7]. Most cases are idiopathic, but some suggested aetiologies include exocrine pancreatic insufficiency, lymphocytic-plasmacytic enteritis and ileocolic carcinoma, intussusception, parvovirus infection, vigorous exercise, closed abdominal trauma, concurrent gastric dilatation volvulus, and gastrointestinal foreign bodies [8] [9] [10] [11]. There are fewer case reports describing partial or segmental small intestinal volvulus in dogs. In two case reports describing localised jejunal volvulus in dogs, the condition was diagnosed two months after surgically treated intussusception or was secondary to intra-abdominal adhesions due to previous gastric foreign body surgery [12] [13].

This case report describes radiographic and ultrasonographic findings in three surgically confirmed cases of small intestinal ischemia related to mesenteric volvulus or intestinal torsion.

\section{Case Report}

\subsection{Cases}

Three dogs presented to the emergency service because of acute vomiting and abdominal discomfort at the Veterinary Teaching Hospital of the University of Helsinki, Finland between 2013 and 2014. Case 1 was an intact 6.5-year-old male Dachshund, case 2 was a 3-month-old intact male Tibetan terrier, and case 3 was a 1.5-year-old intact female Bichon frise. Case 1 and 2 had experienced symptoms approximately 6 and 15 hours respectively before presenting to the hospital. Case 3 had already started vomiting two days prior and had visited a veterinarian on the previous day. Supportive treatment, and an antiemetic drug had been given to the dog, but vomiting continued and the general condition of the dog deteriorated. All dogs had vomited several times and Case 3 also had hematochezia. The owner of the Dachshund reported that the dog had a tendency to chew toys and some other foreign objects. However, none of the dogs had undergone previous surgical procedures related to small intestinal obstructions. The owner of the Dachshund also reported that the dog had lost some weight during the previous months, but otherwise all three dogs had been reported to be generally healthy before the acute episode of vomiting.

In clinical presentation, all dogs showed signs of hypovolemic shock. They were very apathetic and tachycardic (heart rate 140 to 190 beats per minute), all 
had pale mucus membranes, prolonged capillary refill timed, and/or weak peripheral pulse. In all three dogs, abdominal palpation was painful, and in two dogs thickened intestinal loops or a mass like structure was felt with abdominal palpation.

\subsection{Imaging Findings}

Laterolateral and ventrodorsal abdominal radiographs were performed for all three dogs. In two dogs (case 1 and 2), radiographic findings included multiple fluid- and gas-filled moderately to severely dilated small intestinal loops (1.9 $2.6 \times$ L5 height) (Figure 1). In both cases the stomach was partly gas-filled but normally positioned and normal in size. There was normal faecal content in the colon. Radiographic findings suggested a segmental ileus of the small intestine, most likely due to mechanical obstruction, e.g. due to a foreign body. However, no radiopaque foreign bodies were detected. Loss of serosal detail was also noted, which was thought to be due to the small amount of intra-abdominal fat or peritoneal effusion. In case 3 , in the ventral part of the abdomen, there were multiple distended intestinal loops that contained numerous small gas bubbles and some material composed of fluid or soft tissue opacity (Figure 2). Based on the findings, either distended small intestinal loops or displaced colon was suspected. The caudal part of the colon was empty. The stomach was gas- and fluid-filled and distended.

A B-mode abdominal ultrasound was performed in dorsal recumbency (Philips iU22, C5-8 curvilinear transducer, Philips Oy Healthcare, Finland) for all three dogs. In all three dogs, ultrasonographic findings included severely dilated fluid-filled small intestinal loops with markedly reduced or absent peristalsis. There was partial loss of wall layering with increased overall echogenicity of the intestinal wall, but with normal to mildly increased wall thickening, up to 4.5 mm (Figure 3(a) and Figure 3(b)) [14]. No blood flow was detected in the affected small intestinal wall when assessed with colour Doppler adjusted for small flow velocity (Figure 4(a) and Figure 4(b)). In all three cases, a moderate

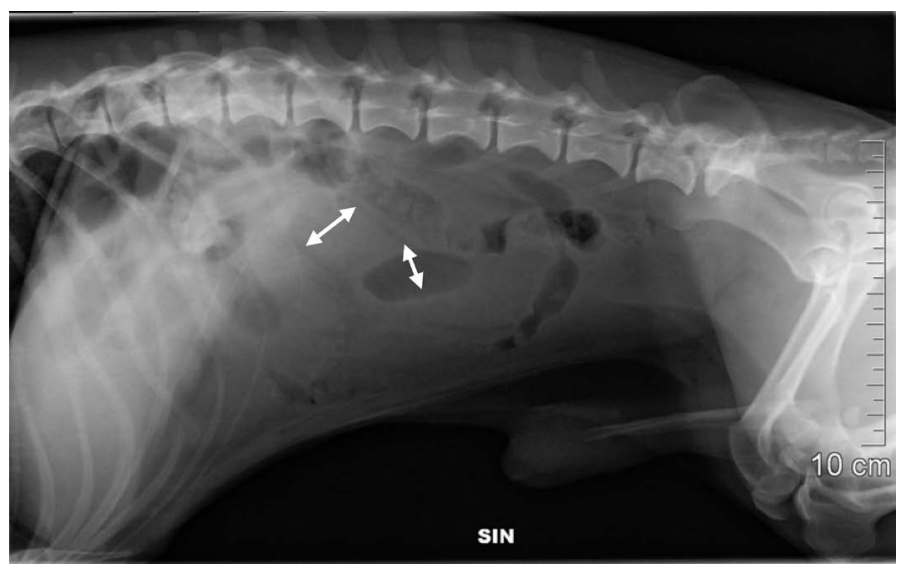

Figure 1. Laterolateral radiograph of case 2 showing multiple distended gas- and fluid-filled small intestinal loops and loss of serosal detail. 


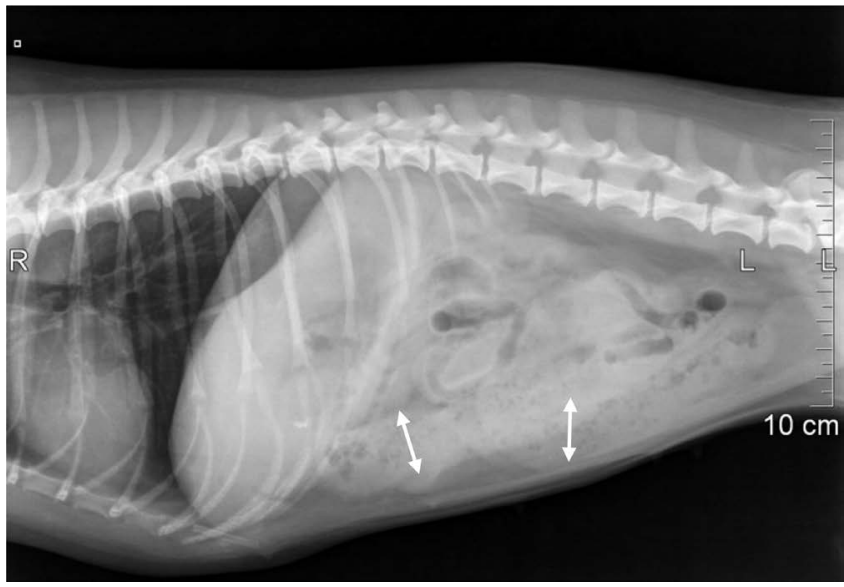

Figure 2. Laterolateral radiograph of case 3 showing multiple distended intestinal loops with numerous small gas bubbles and some material of fluid or soft tissue opacity in the ventral part of the abdomen.

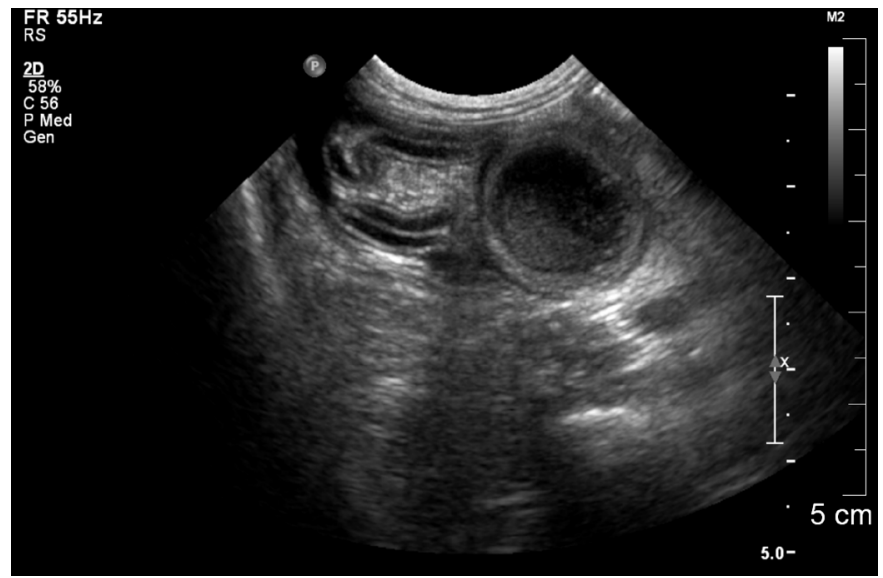

(a)

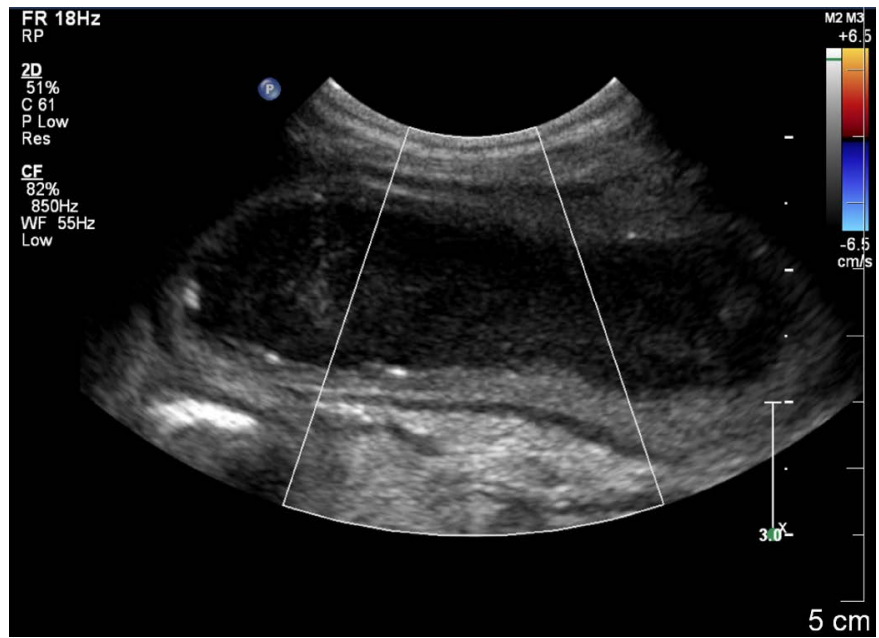

(b)

Figure 3. (a) Transverse ultrasound image of case 2 showing severely dilated small intestinal loop with partial loss of wall layering and increased overall echogenicity of the intestinal wall. Next to the dilated loop there is normal looking small intestine. (b) Longitudinal image of a same affected small intestinal loop as in Figure 3(a). 


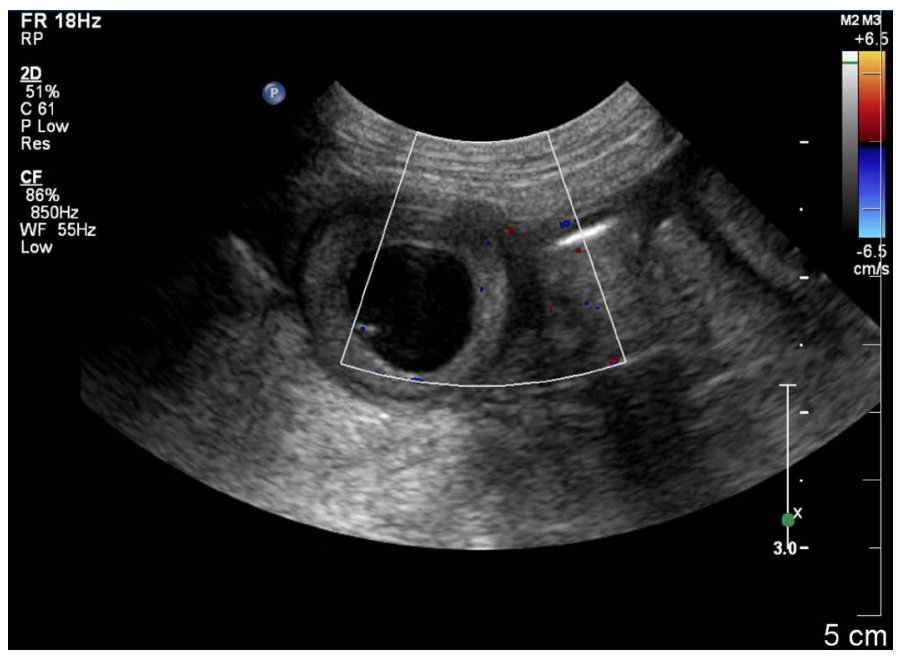

(a)

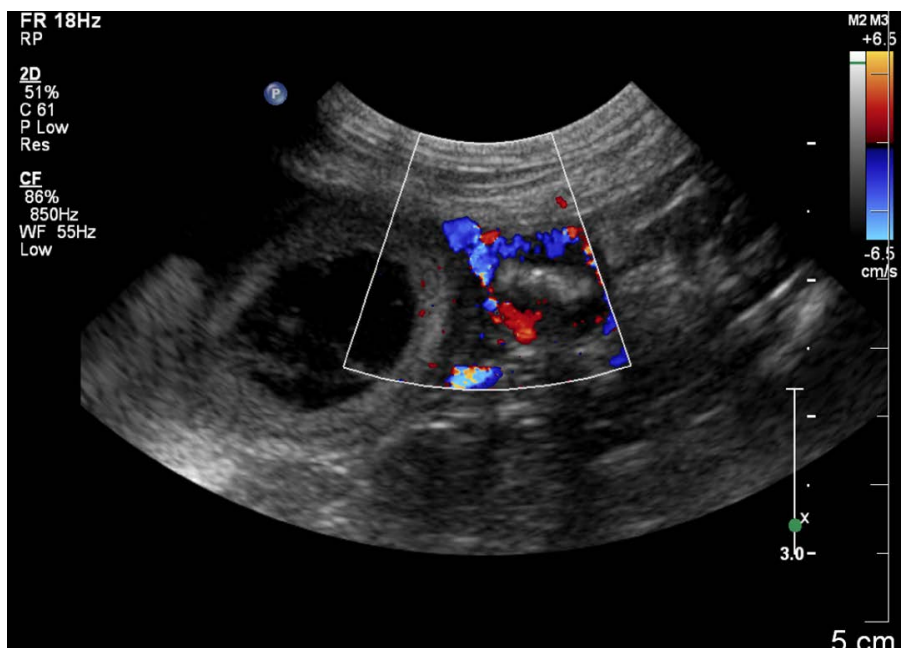

(b)

Figure 4. (a) Transverse image of the affected small intestinal loop of case 3 showing no signal on Doppler; (b) Compared with normal looking adjacent segment of small intestine showing clear Doppler signal.

amount of peritoneal effusion was detected with hyperechoic omental and mesenteric fat tissue throughout the peritoneal cavity. In all dogs, part of the small intestine including the proximal duodenum was normal or only slightly dilated in diameter and showed normal wall layering. There were no findings to support mechanical obstruction due to foreign body. In addition to findings described above in case 3 the affected small intestinal loops contained small gas bubbles and some strand-like structures of soft tissue echogenicity. The location of the small gas bubbles varied and in some parts of the affected small intestine they were suspected to be in the wall of the intestine rather than just in the lumen (Figure 5). Based on ultrasonographic findings an ischemic disease of the small intestine such as mesenteric volvulus or intestinal torsion was suspected. Other differentials included other causes for ischemia (thrombosis) and severe inflammatory changes. 


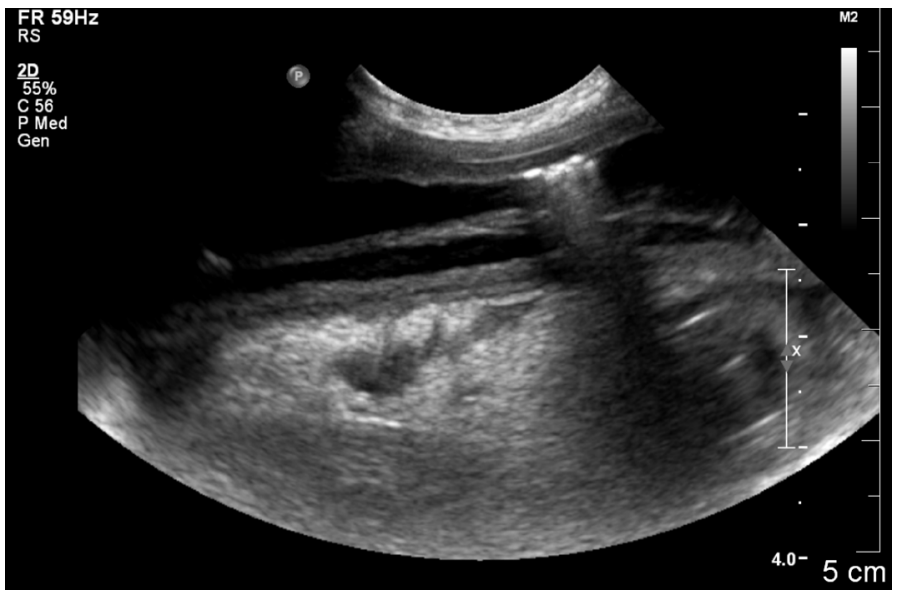

Figure 5. Longitudinal image of the affected small intestine in case 3 showingsmall gas bubbles within or adjacent to the wall of the small intestinal segment and some strand-like structures of soft tissue echogenicity in the lumen of the intestine.

\subsection{Outcome}

Exploratory laparotomy under general anesthesia was performed for all three cases. In case 1, the laparotomy revealed partial volvulus of the small intestine. Approximately half of the jejunum and whole ileum seemed necrotic when observed macroscopically. Intestinal resection was performed and half of the jejunum, ileum, ileocolic junction, and cecum were removed and the jejunum was joined to the colon. In case 2, the main finding of the laparotomy was a $20 \mathrm{~cm}$ segment of dark-coloured small intestine at the area of mid jejunum, that had rotated around itself on its long axis. The affected jejunal segment was resected. In case 3 , the exploratory laparotomy revealed a dark-red to almost-black segment of small intestine. With further evaluation it was noticed that about $2 / 3$ of the jejunum and the whole ileum were twisted on the mesenteric attachment and appeared necrotic. Intestinal resection was performed and the affected part of small intestine, the ileocolic junction, and cecum were removed. In all three cases, a moderate amount of peritoneal free fluid was noted but no clear signs of peritonitis were detected.

All dogs received postsurgical supportive care in the intensive care unit. Case 2 and 3 recovered from surgery without complications and were discharged from the hospital the next day. Despite receiving intensive care, the condition of case 1 deteriorated the next day and the owner elected for euthanasia of the dog. In case 3, diarrhoea started soon after surgery and persisted despite dietary modifications. With tylosin medication (Tylosin tartrate, $120 \mathrm{mg}$, Yliopiston Apteekki, Finland), diarrhoea stopped but reoccurred when medication was tapered down and stopped. Tylosin-responsive diarrhoea was suspected and tylosin medication was continued with the lowest possible dose continuously [15] [16].

\section{Discussion}

There are few reports describing ultrasonographic findings in ischemic diseases 
of small intestine in small animals [6] [17] [18] [19]. In humans, ultrasonography has been used to detect mesenteric volvulus, especially in infants [20]. A whirl sign in which the superior mesenteric vein and mesentery wind around the superior mesenteric artery in a clockwise direction has a good predictive value for midgut volvulus in children with congenital midgut malrotation [20] [21]. Other potential ultrasonographic findings of mesenteric ischemia due to various causes include ileus, reduced or absent intestinal peristalsis, and peritoneal effusion. The appearance of the intestinal wall may be altered due to intestinal wall oedema, the mucosa could change from anechoic to hyperechoic, and submucosa from hyperechoic to hypoechoic [22]. As the condition progresses, intramural or intra-abdominal gas might occur [22]. Intestinal wall ischemia has been evaluated by using colour Doppler ultrasound in humans. Absent or decreased flow signal has been related to intestinal wall ischemia compared with increased flow signal in inflammatory bowel diseases [23] [24]. However, Colour Doppler ultrasonography has some limitations in detecting blood flow, especially in small and slow-flowing vessels [25]. Contrast-enhanced ultrasonography (CEUS) has also been successfully used to diagnose intestinal wall ischemia in humans [26] [27]. However, CEUS is not routinely used in veterinary practice and its availability is limited to more advanced diagnostic imaging units. Computed tomography (CT) with contrast medium is the gold standard for diagnosing acute mesenteric ischemia in humans [22]. Intestinal ischemia can be caused by various conditions such as arterial occlusion, venous occlusion, strangulating obstruction, hypoperfusion associated with non-occlusive vascular disease, ischemia-reperfusion injury, and ischemic colitis each presenting a characteristic CT appearance that aids correct diagnosis in humans [22] [28]. Possible CT findings in small intestinal volvulus include segmental or generalized dilatation of small intestine, intestinal wall thickening, halo appearance and hyperaemia of the intestinal wall, whirl sign, beak-like appearance of the small intestine and closed loops. The abnormal courses of superior mesenteric artery and vein can also be detected [29].

In dogs, mesenteric volvulus or intestinal torsion is usually diagnosed based on clinical findings, radiography and exploratory laparotomy [3] [4] [30] [31]. However, especially in a case of partial mesenteric volvulus or intestinal torsion radiographs can have non-specific findings. This was noted also in our three cases as a failure to reach conclusive diagnosis based on radiographs. In a case of localised mid-jejunal volvulus, abdominal radiographs showed segmental dilatation of the small intestine and loss of serosal detail which are similar findings as in our cases 1 and 2 [12]. Segmental jejunal entrapment, volvulus and strangulation were described in a Dachshund and also in this case similar findings were recorded. Radiographs showed dilated stomach and small intestine with moderate amount of gas and fluid content and mild loss of serosal detail [13]. In complete mesenteric volvulus distended gas-filled small intestinal loops lying parallel to each other can be observed [3]. 
The ultrasonographic features of small intestinal entrapment without using Doppler was described after abdominal surgery in five dogs [17]. Similar findings compared to cases in our study were found. In four dogs, a focal non-motile small intestinal loop with surrounding hyperechoic mesenteric fat was noted. In one of the dogs, the intestinal wall was thickened and corrugated, and in one dog the wall layering of the affected small intestine appeared indistinct. A local accumulation of free fluid around the affected small intestine or generalised abdominal effusion was observed in four dogs [17]. Ultrasound was used to diagnose mesenteric infarction and jejunal necrosis in a cat [18]. Ultrasonographic findings were partly similar as in our cases but appeared clearly later. At initial ultrasound examination, there was segmental small intestinal dilatation with mildly thickened intestinal wall but preserved wall layering. Similar findings were recorded in follow-up ultrasound examination 24 hours later. However, 48 hours after initial presentation ultrasound examination demonstrated clearly thickened intestine wall with loss of wall layering. The wall was hypoechoic which was contradictory to our cases; but a hyperechoic mesentery and small amount of peritoneal free fluid was noticed around the affected small intestinal loop, which was in agreement with our findings [18]. Radiographic and ultrasonographic findings related to mesenteric torsion were described in a 1.5-year-old crossbreed dog [6]. Recorded findings were similar as in our cases, however, in ultrasound examination Doppler mode was not used. Abdominal radiographs showed gas-filled and moderately distended loops of small intestine and loss of serosal detail. Ultrasound examination demonstrated distended gas- and fluid-filled small intestinal loops without peristaltic movement and peritoneal effusion [6]. In some cases of prolonged vascular compromise, necrosis of the intestinal wall and sloughing of the mucosa can lead to thinning of the intestinal wall and amorphous echogenic contents of the intestinal lumen [32].

CT imaging findings of multiphase contrast-enhanced CT have been described in a single dog with mesenteric volvulus including whirl sign, contrast enhancement of the arterial and venous vasculature of the small intestine, moderate contrast enhancement of the small intestine, engorgement of and thrombus formation within jejunal veins, moderate fluid distension of the small intestine, small volume of peritoneal fluid, and left sided displacement of the cecum and ascending colon [19]. Similarly, a whirl sign was also observed in a German shepherd dog with ileocecocolic volvulus [33]. In both cases, abdominal radiography and ultrasonography were inconclusive for volvulus. In the case of mesenteric volvulus, however, ultrasound examination done prior to CT revealed marked distension of the small intestine with reduced definition of wall layering, hypomotility, and a small amount of peritoneal effusion [19]. Potential nephrotoxicity, the risk of an allergic reaction to the contrast agent, and need of general anaesthesia or sedation, limit the use of CT in critically ill patients [22].

In these three cases the onset of clinical symptoms varied between 6 to 48 hours. Acute onset of the clinical signs in case 1 and 2 correlates with previous 
publications [2] [3] [4]. Case 3 had had clinical signs already for two days and at the time of presentation to the hospital the general condition of the dog was clearly worse than in other two cases. This suggests that the duration of the vascular blockage was longer than in other two cases. On the other hand, in this dog the recovery after surgery was uneventful and intraoperatively there were no signs of peritonitis due to prolonged vascular compromise. Therefore, the duration of the volvulus is difficult to determine and might not be as long as the time elapsed from the onset of clinical signs. All three cases presented typical clinical signs previously reported in literature including acute vomiting, signs of hypovolemic shock and painful abdominal palpation [2] [3] [4]. Unlike what has been previously published as suggested, mesenteric or intestinal torsion has been also noted in small breed dogs. In our cases two of the dogs were small breeds, and one was a puppy. No predisposing factors were identified in these dogs. However, because of the possible weight loss reported by the owner in case 1, an underlying chronic illness cannot be completely ruled out as necropsy was not performed after euthanasia. In case 3 diarrhoea started soon after surgery as possible association with intestinal resection. Histological examination of the intestine was not performed and thus potential underlying intestinal disease cannot be excluded.

In intestinal volvulus, the thin walled veins and lymphatics are usually obstructed first, leading to oedema of the intestinal wall [1]. Eventually the mesenteric artery and its branches are obstructed, resulting in necrosis of the intestinal wall [1]. The severity of ultrasonographic changes most likely correlates with the severity and duration of the vascular blockage. In all three of our cases, the affected small intestinal wall was hyperechoic and there was loss of layering. This is most likely due to oedema, hemorrhage and necrosis of the intestinal wall [22] [32]. In case 3 there were strand-like structures in the lumen of the intestine and small gas bubbles within or adjacent to the wall of the intestine. In this case, the dog had had symptoms already for two days and most likely the duration of the vascular blockage was longer than in other two cases. Therefore, the strand-like structures and small gas bubbles most likely represent disruption of the mucosal barrier and sloughing of the mucosa due to vascular compromise [32]. In all three cases the affected small intestine was severely dilated and no peristalsis was observed in this part of the intestine. In these three cases the proximal part of the gastrointestinal tract was normal or only slightly dilated which is opposite to typical mechanical obstruction (e.g. due to foreign body) in which the gastrointestinal tract proximal to obstruction shows the most severe changes. No Doppler signal was present in the examined affected small intestinal loops compared with non-affected loops presenting clearly detectable blood flow. The limitations of colour Doppler ultrasonography such as false signal due to motion artefact should be taken into consideration and multiple sites of the affected intestine should be examined. In addition to changes observed in intestinal wall, a whirl sign can be used to help diagnosing mesenteric volvulus. However, it was not 
visualized in our cases. In all three ultrasound examinations a moderate amount of peritoneal effusion with hyperechoic mesenteric and omental fat tissue was noted. The fluid could represent septic or non-septic exudate due to inflammation and increase in mesothelial and endothelial permeability [34]. The biochemical and cytological analysis of the peritoneal fluid was not performed, however, in the exploratory laparotomy no clear macroscopic signs of peritonitis were observed in any of the dogs.

\section{Conclusion}

In the three described cases, ultrasound was helpful in demonstrating uniform intestinal wall changes and reduced or absent blood flow in the intestinal wall. Our findings strongly suggest that mesenteric volvulus or intestinal torsion needs to be included in the differential diagnosis list of acute abdomen in dogs of all sizes and ages. In the case of disrupted blood circulation, a fast surgical intervention is required to increase patient survival. In addition to radiographs and $\mathrm{CT}$, ultrasonography can provide a quick and noninvasive technique to source additional information and to reach correct diagnosis without need for sedation or general anaesthesia.

\section{Acknowledgements}

The authors are grateful to DVM Susanne Kilpinen for her comments, especially concerning the Outcome chapter.

\section{References}

[1] Brown, D.C. (2003) Small intestine. In: Slatter, D., Ed., Textbook of Small Animal Surgery, 3rd Edition, WB Saunders Co, Philadelphia, 660-661.

[2] Shealy, P.M. and Henderson, R.A. (1992) Canine Intestinal Volvulus: A Report of Nine New Cases. Veterinary Surgery, 21, 15-19. https://doi.org/10.1111/j.1532-950X.1992.tb00005.x

[3] Junius, G., Appeldoorn, A.M. and Schrauwen, E. (2004) Mesenteric Volvulus in the Dog: A Retrospective Study of 12 Cases. Journal of Small Animal Practice, 45, 104-107.https://doi.org/10.1111/j.1748-5827.2004.tb00211.x

[4] Cairó, J., Font, J., Gorraiz, J., Martin, N. and Pons, C. (1991) Intestinal Volvulus in Dogs: A Study of Four Clinical Cases. Journal of Small Animal Practice, 40, 136-140.https://doi.org/10.1111/j.1748-5827.1999.tb03058.x

[5] Spevakow, A.B., Nibblett, B.M., Carr, A.P. and Linn, K.A. (2010) Chronic Mesenteric Volvulus in a Dog. The Canadian Veterinary Journal, 51, 85-88.

[6] Rahal, S.C., Garib, M.I., Mamprim, M.J. and Teixeira, C.R. (2000) Mesenteric Torsion in a Dog. The Canadian Veterinary Journal, 41, 710-711.

[7] Knell, S.C., Andreoni, A.A., Dennler, M. and Venzin, C.M. (2010) Successful Treatment of Small Intestinal Volvulus in Two Cats. Journal of Feline Medicine and Surgery, 12, 874-877. https://doi.org/10.1016/j.jfms.2010.09.007

[8] Westermarck, E. and Rimaila-Pärnänen, E. (1989) Mesenteric Torsion in Dogs with Exocrine Pancreatic Insufficiency: 21 Cases (1978-1987). Journal of the American Veterinary Medical Association, 195, 1404-1406. 
[9] Harvey, H.J. and Rendano, V.T. (1984) Small Bowel Volvulus in Dogs, Clinical Observations. Veterinary Surgery, 13, 91. https://doi.org/10.1111/j.1532-950X.1984.tb00767.x

[10] Nemzek, J.A., Walshaw, R. and Hauptman, J.G. (1993) Mesenteric Volvulus in the Dog: A Retrospective Study. Journal of the American Animal Hospital Association, $29,357$.

[11] Matushek, K.J. and Cockshutt, J.R. (1987) Mesenteric and Gastric Volvulus in a Dog. Journal of the American Veterinary Medical Association, 191, 327-328.

[12] Jasani, S., House, A.K. and Brockman, D.J. (2005) Localised Mid-Jejunal Volvulus Following Intussusception and Enteroplication in a Dog. Journal of Small Animal Practice, 46, 398-401. https://doi.org/10.1111/j.1748-5827.2005.tb00337.x

[13] Di Cicco, M.F., Bennett, R.A., Ragetly, C. and Sippel, K.M. (2011) Segmental Jejunal Entrapment, Volvulus, and Strangulation Secondary to Intra-Abdominal Adhesions in a Dog. Journal of the American Animal Hospital Association, 47, 31-35. https://doi.org/10.5326/JAAHA-MS-5516

[14] Delaney, F., O’Brien, R.T. and Waller, K. (2003) Ultrasound Evaluation of Small Bowel Thickness Compared to Weight in Normal Dogs. Veterinary Radiology and Ultrasound, 44, 577-580. https://doi.org/10.1111/j.1740-8261.2003.tb00510.x

[15] Kilpinen, S., Spillmann, T., Syrjä, P., Skrzypczak, T., Louhelainen, M. and Westermarck, E. (2011) Effect of Tylosin on Dogs with Suspected Tylosin-Responsive Diarrhea: A Placebo-Controlled, Randomized, Double-Blinded, Prospective Clinical Trial. Acta Veterinaria Scandinavica, 53, 26. https://doi.org/10.1186/1751-0147-53-26

[16] Kilpinen, S., Spillmann, T. and Westermarck, E. (2014) Efficacy of Two Low-Dose Oral Tylosin Regimens in Controlling the Relapse of Diarrhea: A Prospective, Single-Blinded, Two-Arm Parallel, Clinical Field Trial. Acta Veterinaria Scandinavica, 56, 43. https://doi.org/10.1186/s13028-014-0043-5

[17] Swift, I. (2009) Ultrasonographic Features of Intestinal Entrapment in Dogs. Veterinary Radiology and Ultrasound, 50, 205-207. https://doi.org/10.1111/j.1740-8261.2009.01518.x

[18] Wallack, S.T., Hornof, W.J. and Herrgesell, E.J. (2003) Ultrasonographic Diagnosis-Small Bowel Infarction in a Cat. Veterinary Radiology and Ultrasound, 44, 81-85. https://doi.org/10.1111/j.1740-8261.2003.tb01454.x

[19] Chow, K.E., Stent, A.W. and Milne, M. (2014) Imaging Diagnosis-Use of Multiphasic Contrast-Enhanced Computed Tomography for Diagnosis of Mesenteric Volvulus in a Dog. Veterinary Radiology and Ultrasound, 55, 74-78. https://doi.org/10.1111/vru.12053

[20] Chao, H.C., Kong, M.S., Chen, J.Y., Lin, S.J. and Lin, J.N. (2000) Sonographic Features Related to Volvulus in Neonatal Intestinal Malrotation. Journal of Ultrasound in Medicine, 19, 371-376. https://doi.org/10.7863/jum.2000.19.6.371

[21] Epelman, M. (2006) The Whirlpool Sign. Radiology, 240, 910-911. https://doi.org/10.1148/radiol.2403040370

[22] Reginelli, A., Genovese, E., Cappabianca, S., Iacobellis, F., Berritto, D., Fonio, P., Coppolino, F. and Grassi, R. (2013) Intestinal Ischemia: US-CT Findings Correlations. Critical Ultrasound Journal, 15, S7. https://doi.org/10.1186/2036-7902-5-S1-S7

[23] Shirahama, M., Ishibashi, H., Onohara, S., Dohmen, K. and Miyamoto, Y. (1999) Colour Doppler Ultrasound for the Evaluation of Bowel Wall Thickening. British Journal of Radiology, 72, 1164-1169. https://doi.org/10.1259/bjr.72.864.10703473

[24] Smereczyński, A., Starzyńska, T. and Kołaczyk, K. (2013) Ultrasound of Selected Pathologies of the Small Intestine. Journal of Ultrasonography, 13, 155-166. 
https://doi.org/10.15557/JoU.2013.0016

[25] Kong, M.S., Wong, H.F., Lin, S.L., Chung, J.L. and Lin, J.N. (1997) Factors Related to Detection of Blood Flow by Color Doppler Ultrasonography in Intussusception. Journal of Ultrasound in Medicine, 16, 141-144. https://doi.org/10.7863/jum.1997.16.2.141

[26] Hamada, T., Yamauchi, M., Tanaka, M., Hashimoto, Y., Nakai, K. and Suenaga, K. (2007) Prospective Evaluation of Contrast-Enhanced Ultrasonography with Advanced Dynamic Flow for the Diagnosis of Intestinal Ischaemia. British Journal of Radiology, 80, 603-608. https://doi.org/10.1259/bjr/59793102

[27] Hata, J., Kamada, T., Haruma, K. and Kusunoki, H. (2005) Evaluation of Bowel Ischemia with Contrast-Enhanced US: Initial Experience. Radiology, 236, 712-715. https://doi.org/10.1148/radiol.2362040299

[28] Furukawa, A., Kanasaki, S., Kono, N., Wakamiya, M., Tanaka, T., Takahashi, M. and Murata, K. (2009) CT Diagnosis of Acute Mesenteric Ischemia from Various Causes. American Journal of Roentgenology, 5, 408-416. https://doi.org/10.2214/AJR.08.1138

[29] Feng, S.T., Chan, T., Sun, C.H., Li, Z.P., Guo, H.Y., Yang, G.Q., Peng, Z.P. and Meng, Q.F. (2010) Multiphasic MDCT in Small Bowel Volvulus. European Journal of Radiology, 76, 13-18.

[30] Halfacree, Z.J., Beck, A.L., Lee, K.C. and Lipscomb, V.J. (2006) Torsion and Volvulus of the Transverse and Descending Colon in a German Shepherd Dog. Journal of Small Animal Practice, 47, 468-470. https://doi.org/10.1111/j.1748-5827.2006.00018.x

[31] Bentley, A.M., O’Toole, T.E., Kowaleski, M.P., Casale, S.A. and McCarthy, R.J. (2005) Volvulus of the Colon in Four Dogs. Journal of the American Veterinary Medical Association, 15, 253-256, 236-237. https://doi.org/10.2460/javma.2005.227.253

[32] Penninck, D. and d'Anjou, M. (2015) Gastrointestinal Tract. In: Penninck, D. and d'Anjou, M., Eds., Atlas of Small Animal Ultrasonography, 2nd Edition, Wiley Blackwell, 272-274.

[33] Javard, R., Specchi, S., Benamou, J., Lapointe, C., Deffontaines, J.B., Planté, J. and d'Anjou, M.A. (2014) Ileocecocolic Volvulus in a German Shepherd Dog. The Canadian Veterinary Journal, 55, 1096-1099.

[34] Dempsey, S.M. and Ewing, P.J. (2011) A Review of the Pathophysiology, Classification, and Analysis of Caninen and Feline Effusions. Journal of the American Animal Hospital Association, 47, 1-11. https://doi.org/10.5326/JAAHA-MS-5558 
Submit or recommend next manuscript to SCIRP and we will provide best service for you:

Accepting pre-submission inquiries through Email, Facebook, LinkedIn, Twitter, etc. A wide selection of journals (inclusive of 9 subjects, more than 200 journals)

Providing 24-hour high-quality service

User-friendly online submission system

Fair and swift peer-review system

Efficient typesetting and proofreading procedure

Display of the result of downloads and visits, as well as the number of cited articles Maximum dissemination of your research work

Submit your manuscript at: http://papersubmission.scirp.org/

Or contact ojvm@scirp.org 